\title{
Macroeconomic effects of bank secrecy when tax evasion is endogenous
}

Frode Brevik and Manfred Gärtner

University of St. Gallen

Switzerland 


\section{The debate on bank secrecy}

- International Institutions and Organizations

OECD, IMF, EU commission

The discussion within the European Union

Austria, Belgium, Luxembourg

Jersey, Dutch Antilles, Madeira, ...

EU - Switzerland

bilateral negotiations

- Non-government organizations

Bank secrecy and developing countries

- Academic work

Boadway and Keen (1998). International Economic Review. 


\section{The scope of positions taken}

The goal is to secure the integrity of tax systems by addressing the issues raised by practices with respect to mobile activities that unfairly erode the tax bases of other countries and distort the location of capital and services. Such practices can also cause undesired shifts of part of the tax burden to less mobile tax bases, such as labor, property, and consumption, ... [OECD (2000), p.5].

Our results ,,may point, .., to a useful social purpose for tax havens". [Boadway and Keen (1998), p. 473] 


\section{Initial questions}

Which type of model appears promising?

Representative Agents, Overlapping Generations, RBC models, ...

- In which macroeconomic context do we analyze the issues?

Small open economy, large open economy, 2-country model, n-country model; perfect competition?

- Exogenous and endogenous variables

Tax payers' honesty (degree of tax evasion), government preferences, international tax cooperation

- How to make tax rates endogenous

Public spending in the utililty function, public spending as a factor of production, income distribution in the utility function 


\section{An $n$-country OLG model with 2 types of countries}

- Firms

operate under perfect competition

$$
\begin{aligned}
y & =k^{\alpha} \\
r & =\alpha k^{\alpha-1}-\delta \\
w & =(1-\alpha) k^{\alpha}
\end{aligned}
$$

- Households

maximize 2-period, lifetime utility

$$
\begin{array}{r}
U=\log c_{y}+\beta \log c_{o}^{\prime}+\psi_{i}(1+\beta) \log g \\
\text { with } i \in\{e u, c h\}
\end{array}
$$

- Government

maximizes 2-period utility, may possess preferences different from households

$$
\begin{array}{r}
U=\log c_{y}+\beta \log c_{o}^{\prime}+\psi_{i}(1+\beta) \log g \\
\text { with } i \in\{e u, c h\}
\end{array}
$$




\section{Effects of bank secrecy}

\section{- Analytically}

possible for some variables, for example:

$$
\begin{aligned}
& \frac{d \log c_{y, e u}}{d t_{e u}}=-\frac{1}{1-t_{\text {eu }}} \\
& \frac{d \log c_{o, \text { eu }}}{d t_{\text {eu }}}=-\frac{(1-f) r}{\bar{t} r+1}+\frac{d f}{d t_{\text {eu }}} \frac{t_{\text {eu }}-t_{f}}{\bar{t} r+1}-\frac{1}{1-t_{\text {eu }}} \\
& \frac{d \log g_{\text {eu }}}{d t_{e u}}=\frac{1}{t_{c h}}-\frac{1}{1-t_{c h}} \frac{T_{c h}^{a}}{g_{c h}}-\frac{d f}{d t} \frac{1}{1-f} \frac{T_{e u}^{a}}{g_{e u}}
\end{aligned}
$$

$$
\begin{aligned}
\frac{d \log c_{y, c h}}{d t_{c h}} & =-\frac{1}{1-t_{c h}} \\
\frac{d \log c_{o, c h}}{d t_{c h}} & =-\frac{1}{1-t_{c h}}-\frac{r}{\left(1-t_{c h}\right) r+1} \\
\frac{d \log g_{c h}}{d t_{c h}} & =\frac{1}{t_{c h}}\left(1-\frac{T_{c h}^{f}}{g_{c h}}\right)-\frac{1}{1-t_{c h}} \frac{T_{c h}^{a}}{g_{c h}}
\end{aligned}
$$

ש Numerically

after calibration and simulation 


\section{Results with exogenous tax evasion}

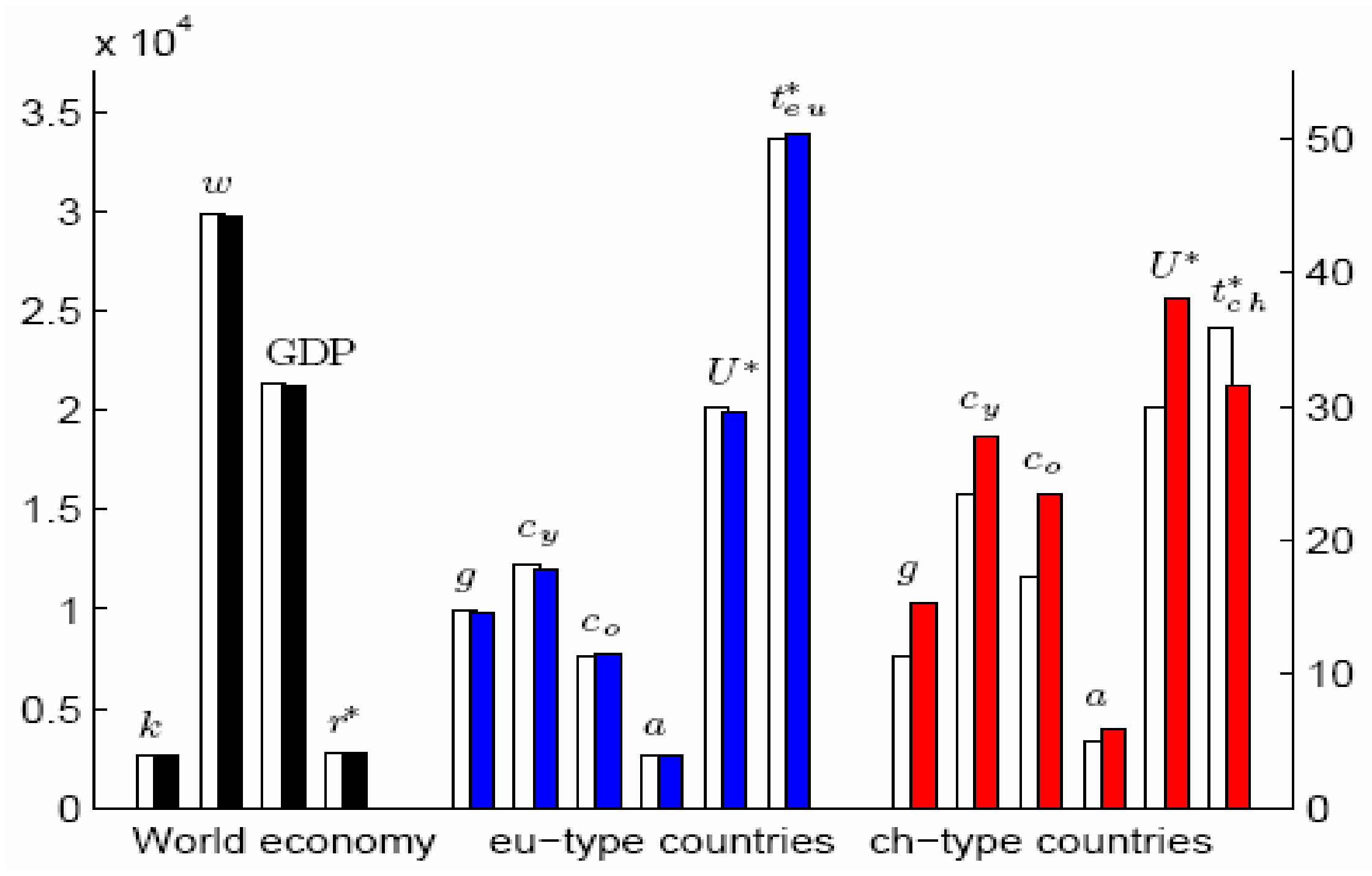

Changes from white to coloured bars indicate the impacts of the introduction of bank secrecy laws 


\section{Initial situation without bank secrecy}

\begin{tabular}{lrrrrrrr}
\hline & \multicolumn{3}{c}{$c h$ countries } & \multicolumn{3}{c}{ eu countries } \\
\cline { 2 - 4 } \cline { 5 - 7 } & $(\mathrm{a})$ & $(\mathrm{b})$ & $(\mathrm{c})$ & $(\mathrm{a})$ & $(\mathrm{b})$ & $(\mathrm{c})$ \\
\hline$t$ & 24.78 & 11.20 & -54.82 & 53.66 & 54.51 & 1.59 \\
& 4,303 & 5,060 & 17.60 & 2,651 & 2,592 & -2.23 \\
$c$ & 17,347 & 21,790 & 25.61 & 9,249 & 9,148 & -1.09 \\
$c_{y}$ & 18,231 & 21,439 & 17.60 & 11,232 & 10,981 & -2.23 \\
$c_{o}$ & 16,463 & 22,140 & 34.49 & 7,266 & 7,314 & 0.66 \\
$g$ & 5,715 & 7,673 & 34.26 & 10,710 & 10,550 & -1.49 \\
$w_{\text {net }}$ & 22,534 & 26,499 & 17.60 & 13,882 & 13,573 & -2.23 \\
$r_{\text {net }}($ annualized $)$ & 3.41 & 3.76 & 10.19 & 2.55 & 2.63 & 2.93 \\
\hline GDP & 385 & 384 & -0.39 & 12,839 & 12,788 & -0.39 \\
GNP & 454 & 487 & 7.35 & 12,770 & 12,685 & -0.67 \\
Labor income & 270 & 269 & -0.39 & 8,987 & 8,952 & -0.39 \\
Capital income & 184 & 219 & 18.69 & 3,783 & 3,733 & -1.32 \\
\hline$U$ & & & 21.96 & & & -1.57 \\
\hline
\end{tabular}




\section{Effect on the global economy}

with

without

bank secrecy change in percent

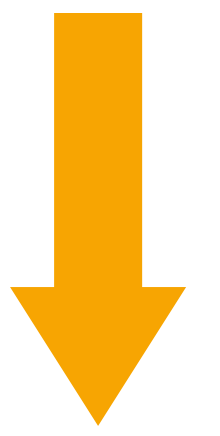

Global economy

\begin{tabular}{lrrr}
\cline { 2 - 4 } & $(\mathrm{a})$ & $(\mathrm{b})$ & $(\mathrm{c})$ \\
\hline$k$ & 2699 & 2663 & -1.31 \\
$w$ & 29958 & 29839 & -0.39 \\
$r$ & 3.98 & 4.00 & 0.60 \\
GDP, GNP & 13224 & 13172 & -0.39
\end{tabular}




\section{Effects on the ch-type economy}

\begin{tabular}{lrrr} 
& \multicolumn{3}{c}{$c h$ countries } \\
\cline { 2 - 4 } & $(\mathrm{a})$ & $(\mathrm{b})$ & $(\mathrm{c})$ \\
\hline$t$ & 24.78 & 11.20 & -54.82 \\
$a$ & 4,303 & 5,060 & 17.60 \\
$c$ & 17,347 & 21,790 & 25.61 \\
$c_{y}$ & 18,231 & 21,439 & 17.60 \\
$c_{o}$ & 16,463 & 22,140 & 34.49 \\
$g$ & 5,715 & 7,673 & 34.26 \\
$w_{\text {net }}$ & 22,534 & 26,499 & 17.60 \\
$r_{\text {net }}$ (annualized) & 3.41 & 3.76 & 10.19 \\
\hline GDP & 385 & 384 & -0.39 \\
GNP & 454 & 487 & 7.35 \\
Labor income & 270 & 269 & -0.39 \\
Capital income & 184 & 219 & 18.69 \\
\hline$U$ & & & 21.96 \\
\hline
\end{tabular}




\section{Effects on the eu-type economy}

\begin{tabular}{lrrr} 
& \multicolumn{3}{c}{ eu countries } \\
\cline { 2 - 4 } & 53.66 & 54.51 & 1.59 \\
$t$ & 2,651 & 2,592 & -2.23 \\
$c$ & 9,249 & 9,148 & -1.09 \\
$c$ & 11,232 & 10,981 & -2.23 \\
$c_{y}$ & 7,266 & 7,314 & 0.66 \\
$c_{o}$ & 10,710 & 10,550 & -1.49 \\
$g$ & 13,882 & 13,573 & -2.23 \\
$w_{\text {net }}$ & 2.55 & 2.63 & 2.93 \\
$r_{\text {net }}($ annualized $)$ & 12,839 & 12,788 & -0.39 \\
\hline GDP & 12,770 & 12,685 & -0.67 \\
GNP & 8,987 & 8,952 & -0.39 \\
Labor income & 3,783 & 3,733 & -1.32 \\
Capital income & & & -1.57 \\
\hline$U$ &
\end{tabular}




\section{Endogenous tax evasion: mechanical approach}

Let tax evasion of eu-citizen depend positively on the tax differential:

$$
f=a+b\left(t_{e u}-t_{f}\right)
$$

The tax elasticity $\varepsilon_{\mathrm{t}}^{f}$ of tax evasion $f$ is defined as

$$
\varepsilon_{t}^{f}=\frac{d f}{f} \frac{t_{e u}-t_{f}}{d t_{e u}}=-\frac{d f}{f} \frac{t_{e u}-t_{f}}{d t_{f}}
$$

\section{Presumption:}

If $f$ is unelastic to the tax rate, the government will raise the tax rate to recapture some of the lost revenues caused by tax evasion.

As $\varepsilon_{\mathrm{t}}^{f}$, increases, raising tax rates become less attractive because higher tax revenues on declared income will be increasingly offset by even more tax evasion.

$\rightarrow$ impact of bank secrecy laws depends on $\varepsilon_{t}^{f}$ 


\section{Mechanical approach: Results (1)}

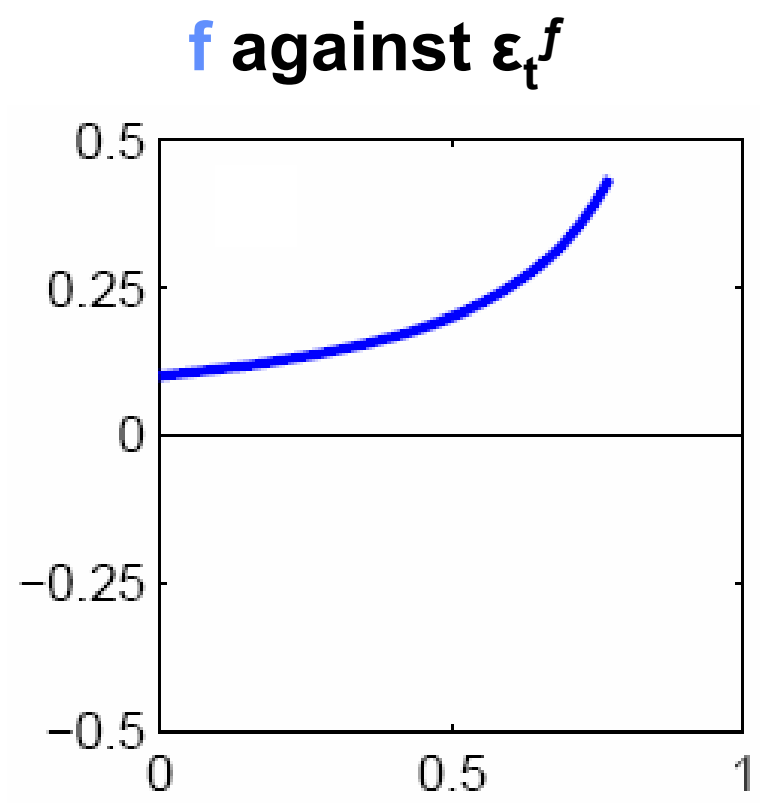

GDP $(\% \Delta)$ against $\varepsilon_{\mathrm{t}} f$

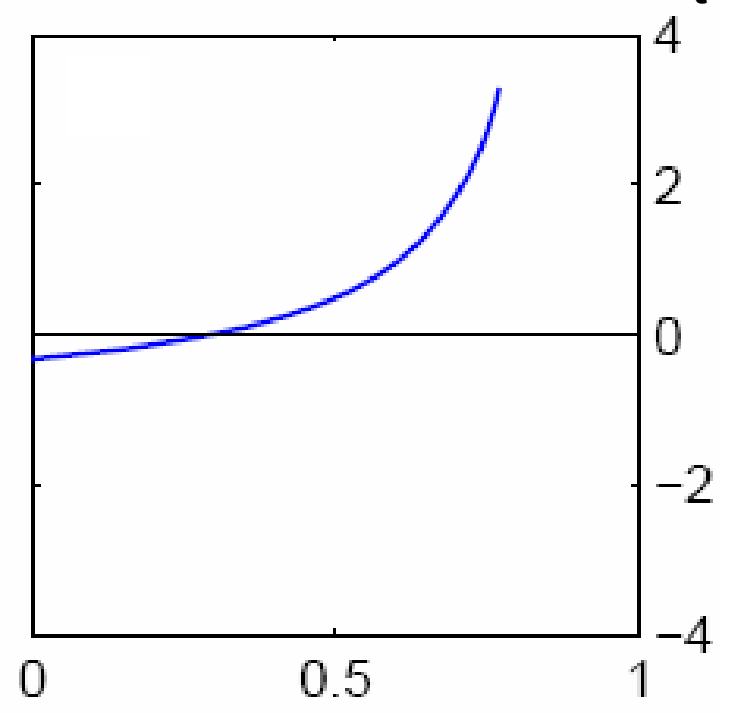

$\mathrm{k}(\% \Delta)$ against $\varepsilon_{\mathrm{t}}^{f}$

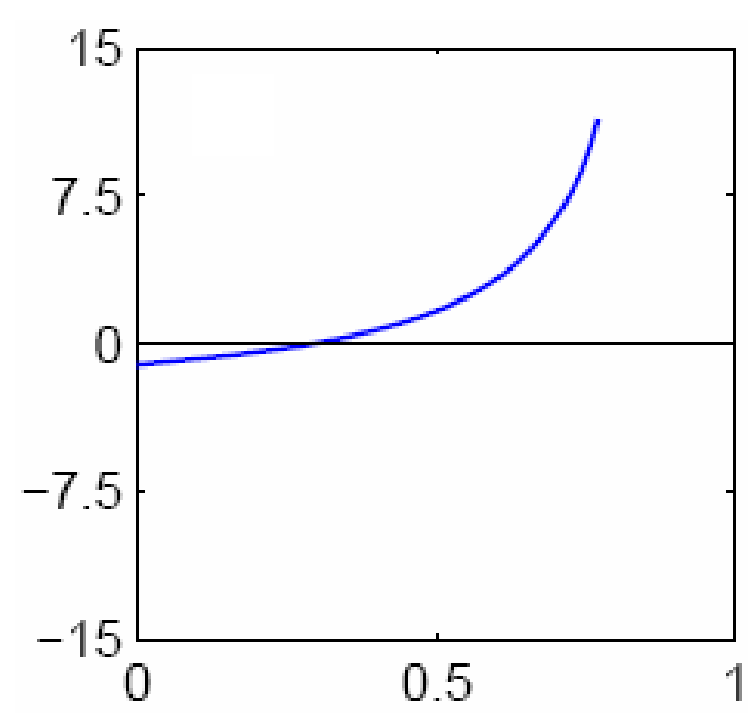

r, w (\% $(\%)$ against $\varepsilon_{\mathrm{t}}^{f}$

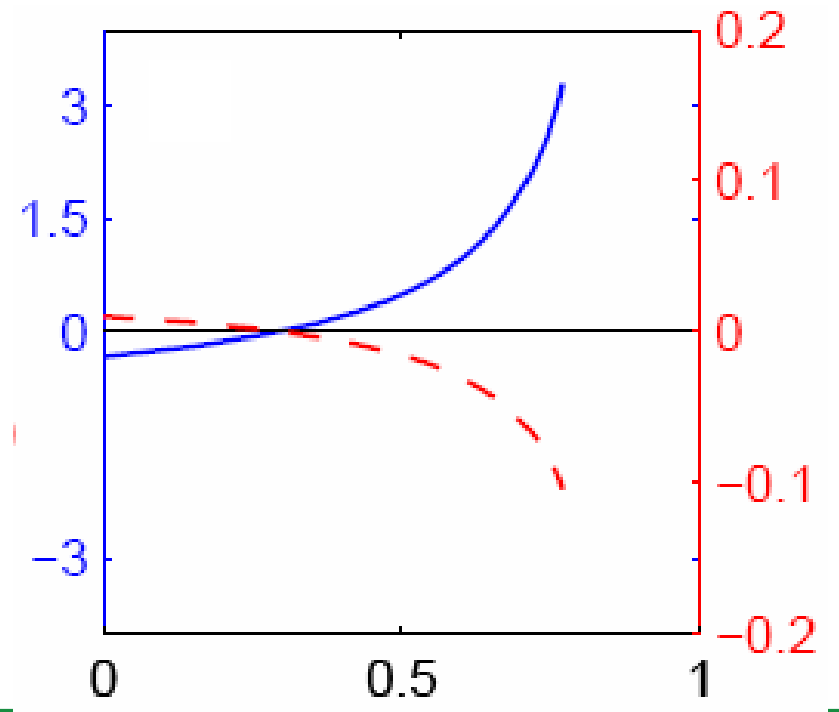

WEAI 82nd Annual Conference, July 1, 2007, slide 13 


\section{Mechanical approach: Results (2)}

t $(\% \Delta)$ against $\varepsilon_{\mathrm{t}}{ }^{f}$

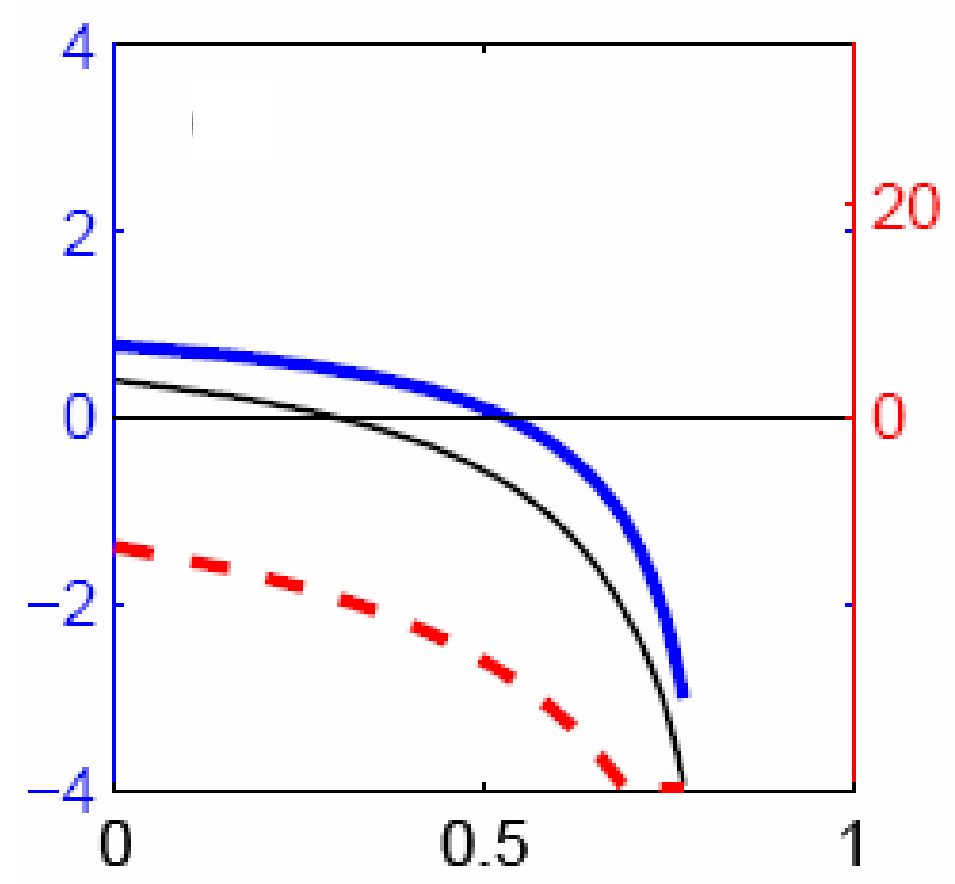

g (\% $\%)$ against $\varepsilon_{\mathrm{t}}{ }^{f}$

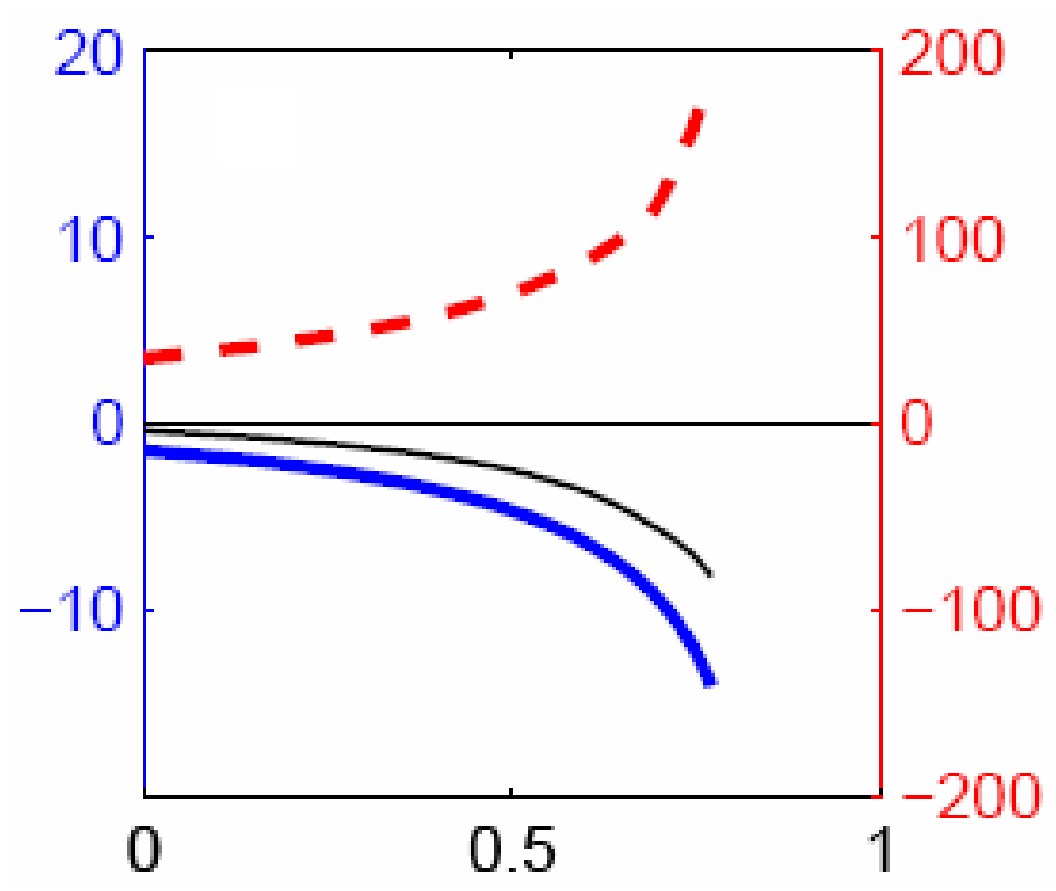

- eu countries

... ch countries

w world 


\section{Mechanical approach: Results (3)}
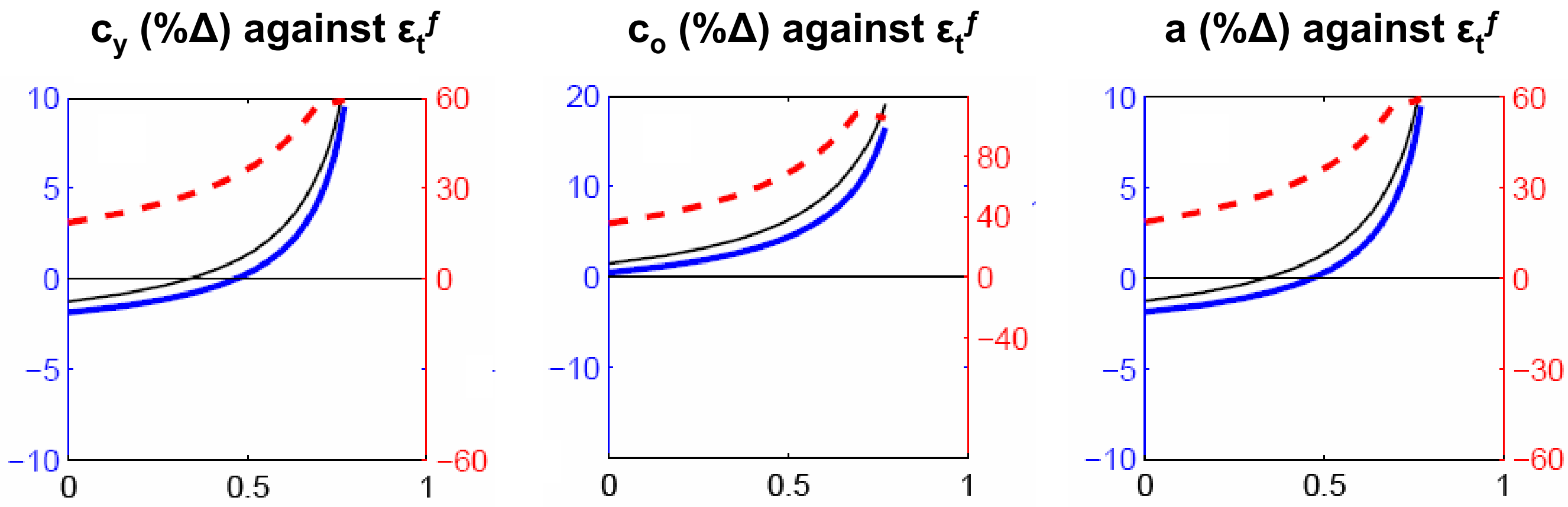

- eu countries

.... ch countries

w world 


\section{Endogenous Tax Evasion: Optimizing Approach (1)}

Household benefit of tax evasion:

$$
B=r\left(t_{e u}-t_{f}\right) f
$$

Household cost of tax evasion:

(i):

$$
C= \begin{cases}C_{0} & \text { if } 0<f \leq f_{1} \\ C_{0}+C_{1}\left(f_{1}-f\right) & \text { if } f_{1}<f \leq f_{2} \\ C_{2} & \text { if } f_{2}<f\end{cases}
$$

(ii):

$$
C= \begin{cases}C_{0} & \text { if } 0<f \leq f_{1} \\ C_{0}+C_{1}\left(f_{1}-f\right)^{2} & \text { if } f_{1}<f \leq f_{2} \\ C_{2} & \text { if } f_{2}<f\end{cases}
$$




\section{Endogenous Tax Evasion: Optimizing Approach (1i)}

\section{Case (i):}
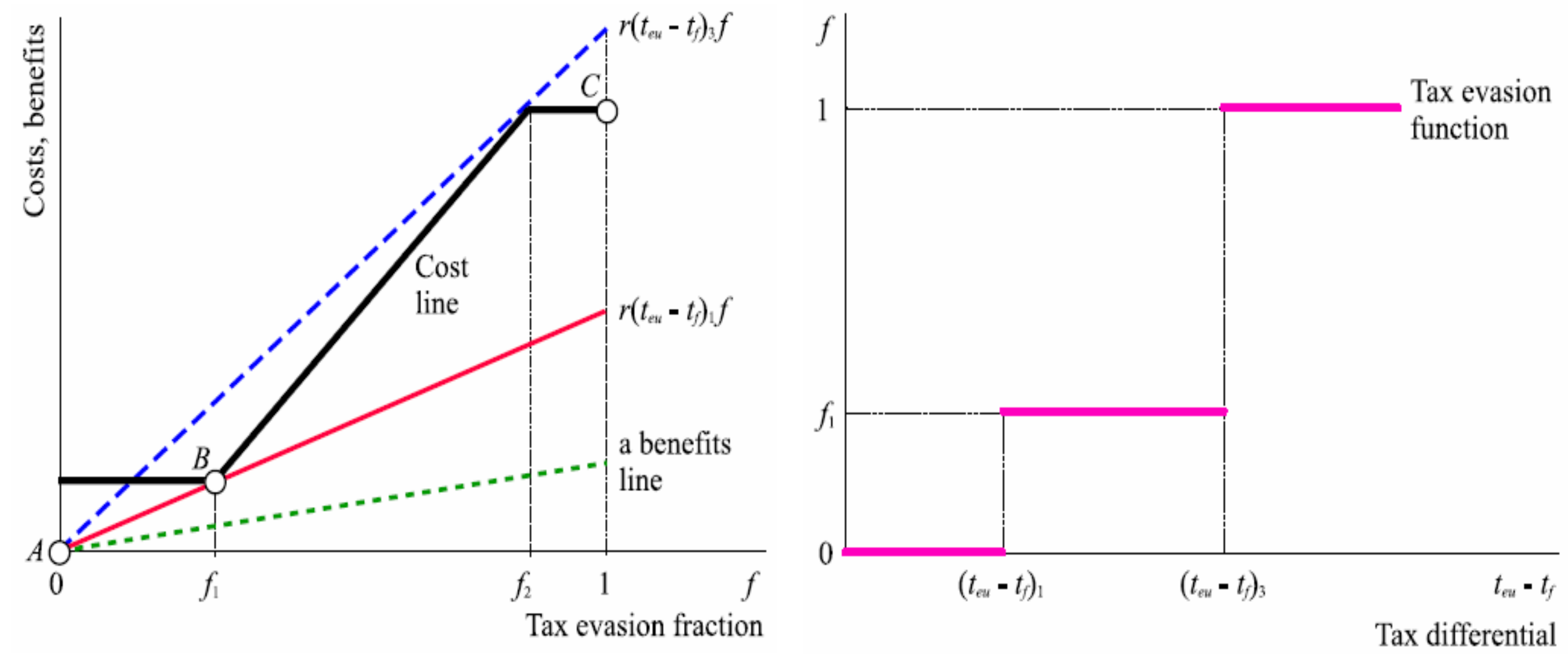

$\rightarrow$ Costs that are linear in $f$ imply a stepwise tax evasion function. This suggests inelastic $f$ with respect to tax differential and thus yields same results as when $f$ is exogenous. 


\section{Endogenous Tax Evasion: Optimizing Approach (1ii)}

Case (ii):
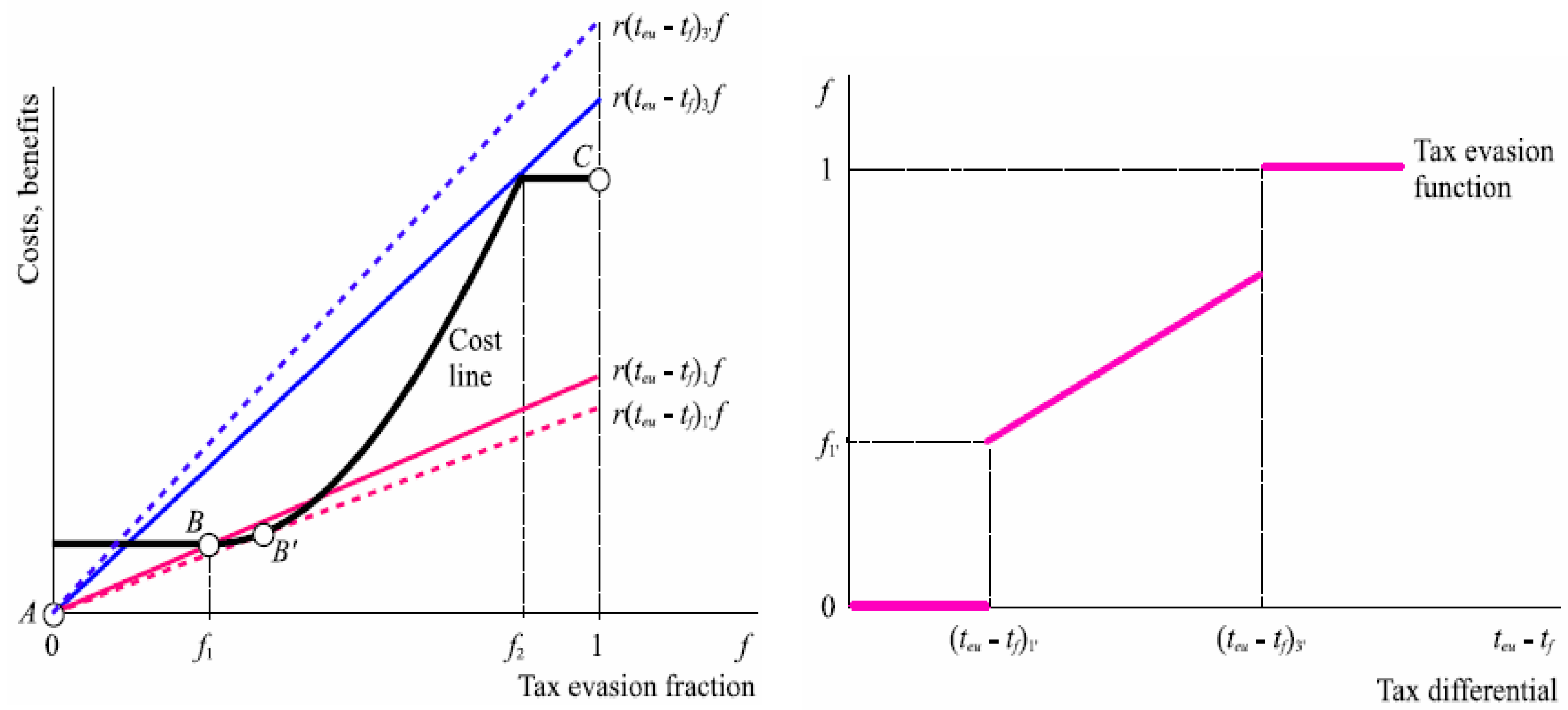

$\rightarrow$ Costs that are quadratic in $f$ imply a linear tax evasion function. This suggests results to be similar to the case when tax evasion was endogenized in a mechanical fashion. 


\section{Endogenous Tax Evasion: Optimizing Approach (2)}

General specification of marginal cost function:

$$
M C=\xi\left(1-\left(1-f^{\eta}\right)^{\theta}\right)
$$

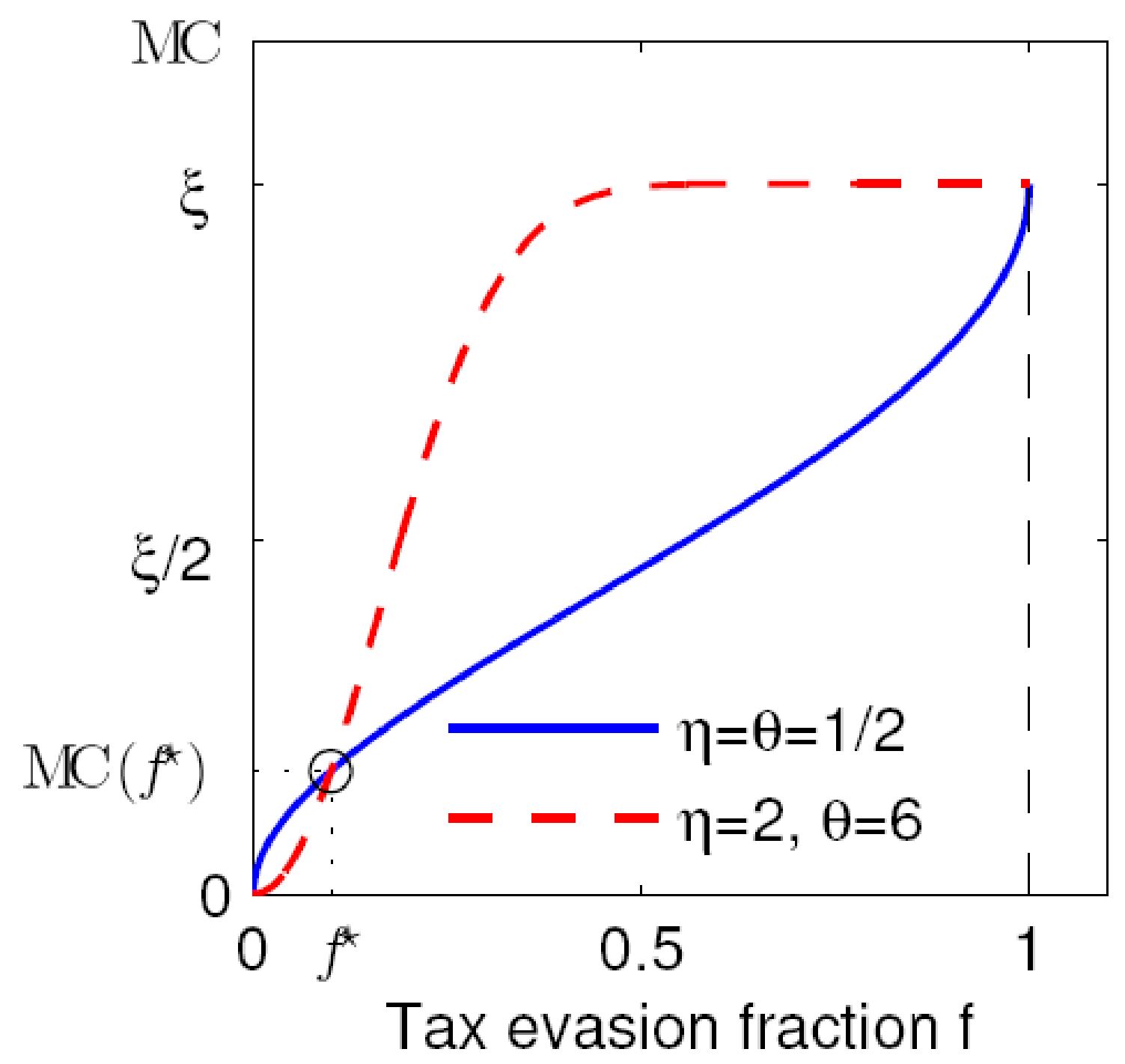




\section{Endogenous Tax Evasion: Optimizing Approach (2)}

General specification of marginal cost function:

$$
M C=\xi\left(1-\left(1-f^{\eta}\right)^{\theta}\right)
$$

Implies following choice of fraction of wealth $f^{*}$ held abroad:

$$
f^{*}=\left\{1-\left[1-\frac{\left(t_{e u}-t_{f}\right) r}{\xi}\right]^{\theta}\right\}
$$

$\rightarrow$ Different parameterizations of marginal cost function generate different tax evasion functions with different tax elasticity $\varepsilon_{\mathrm{t}}^{f}$ at targeted point of intersection. 


\section{Endogenous Tax Evasion: Optimizing Approach (2)}

General specification:

$$
M C=\xi\left(1-\left(1-f^{\eta}\right)^{\theta}\right)
$$

Fraction of wealth $f^{*}$ held abroad:

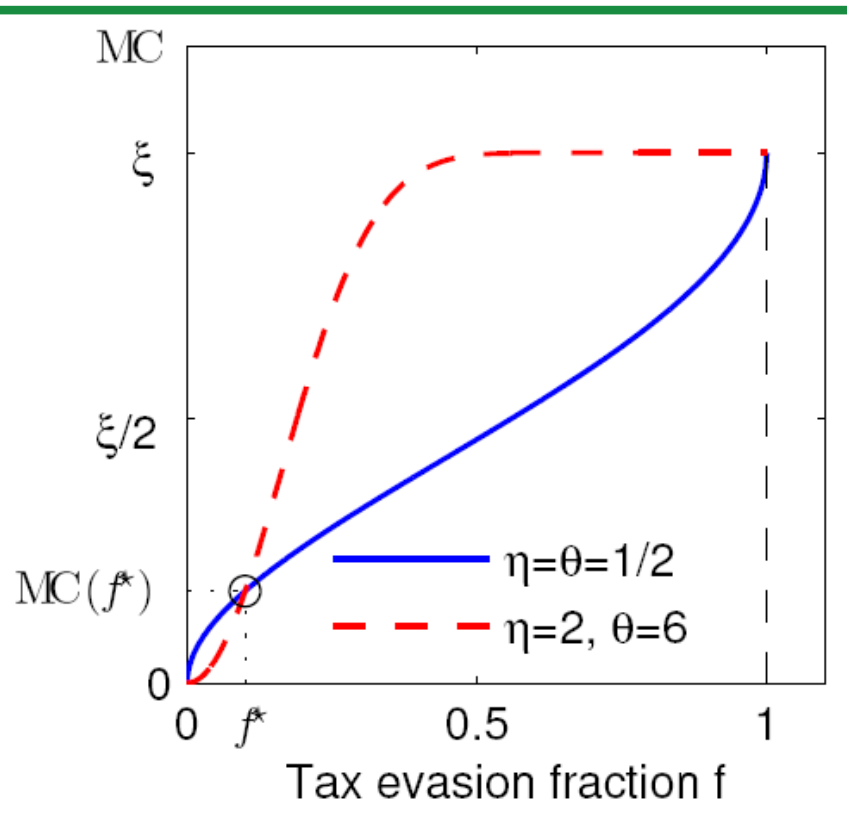

$$
f *=\left\{1-\left[1-\frac{\left(t_{\text {eu }}-t_{f}\right) r}{\xi}\right]^{\theta}\right\}
$$

Model is calibrated so that all $f$ lines intersect at $f=0.1$. All $M C$ functions yield the same share of assets held abroad, but different tax elasticites.

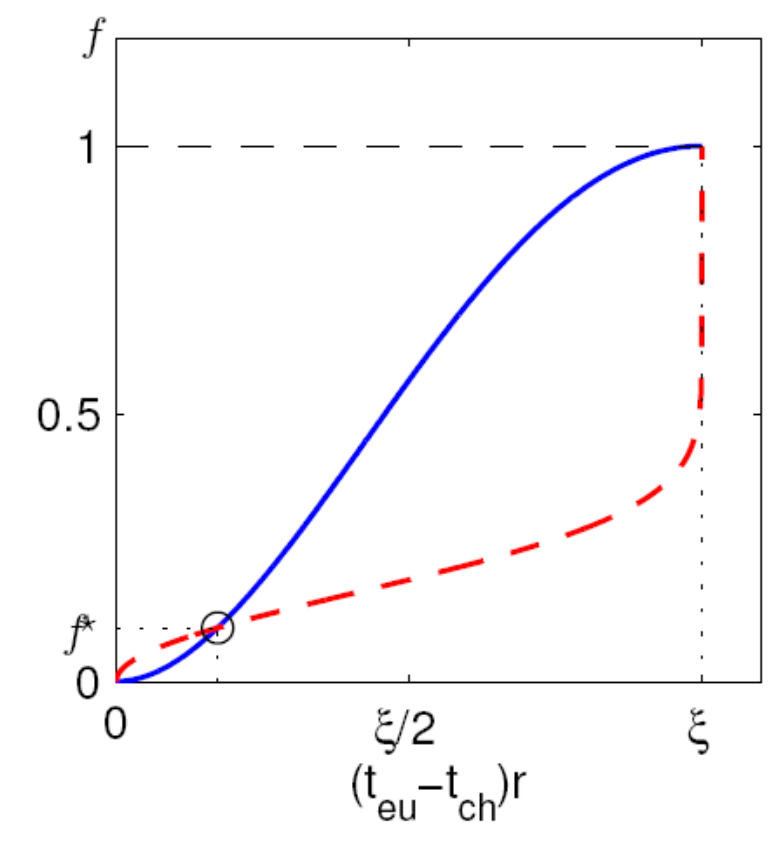




\section{Endogenous Tax Evasion: Optimizing Approach (2a)}

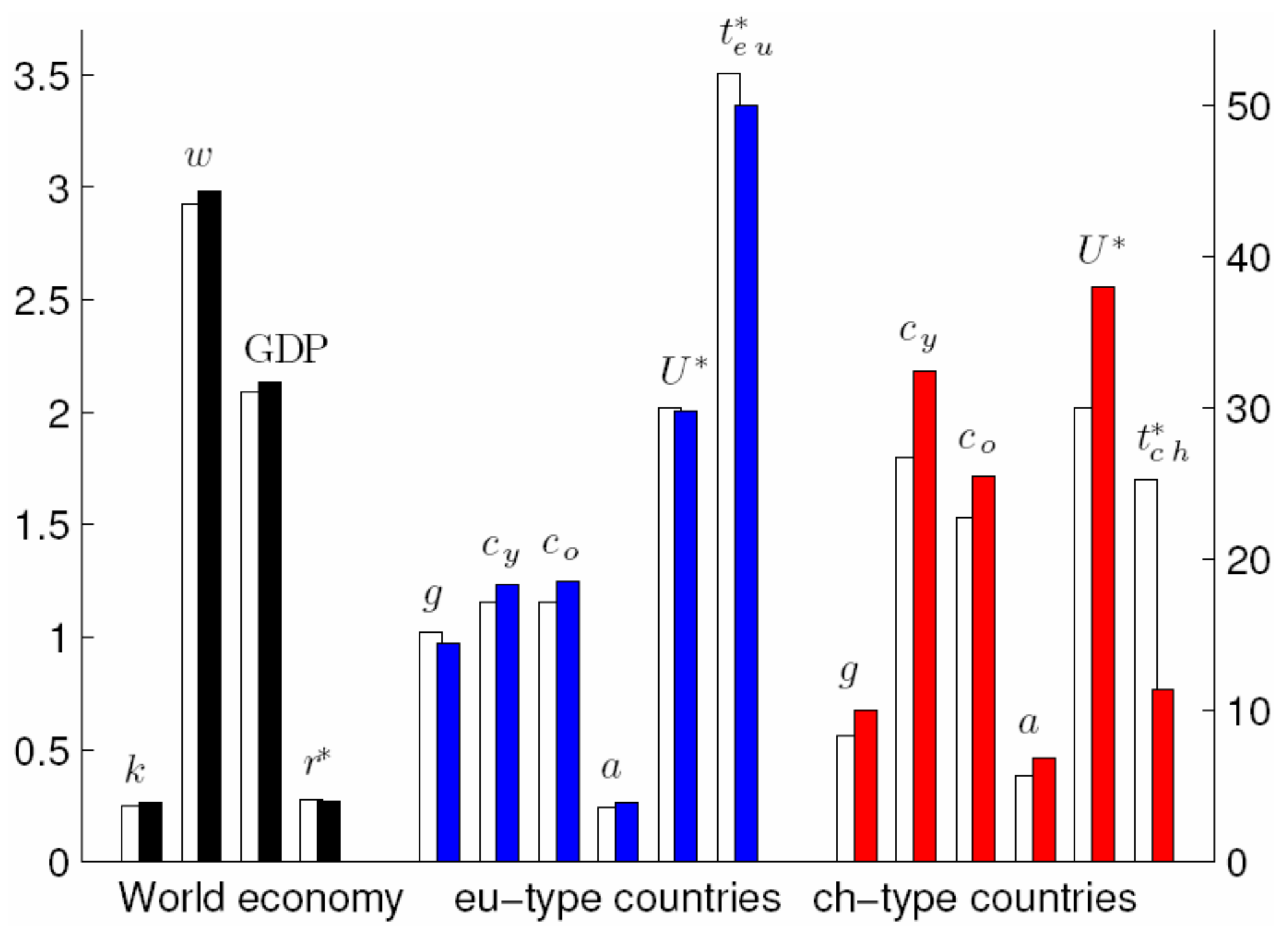

White bars show outcomes with bank secrecy laws Colored bars show outcomes with bank secracy laws 


\section{Main Results}

- When the tax evasion is exogenous or inelastic with respect to tax differentials, the introduction of bank secrecy laws has the following consequences:

- ch-type countries benefit from lower tax rates, more public goods, as well as higher income, wealth and consumption.

- eu-type countries pay for this through taxe rates, a lower level of public goods, as well as lower income, wealth and consumption of the young.

- The effect of banking secrecy on eu-type countries changes, however, when tax evasion exceeds a critical threshhold:

- then tax rates fall, income consumption and wealth increase, but public goods provision deteriorates.

- However: Utility in eu-type countries still drops!

- Independent of $\varepsilon_{\mathrm{t}}^{f}$, eu-type countries' welfare always suffers because

- lower tax rates mean that public spending now falls short of desired level.

- part of paid taxes go to foreign governments, which widens this gap.

- part of the cost of tax evasion is dead-weight loss, not generating utility. 